\title{
LOS PRINCIPIOS DE LA DOCTRINA SOCIAL DE LA IGLESIA EN EL PROYeCto EDUCATIVO institucional de la Uladech Católica
}

\author{
THE PRINCIPLES OF THE SOCIAL DOCTRINE OF THE CHURCH IN \\ institutional Educational Project at Uladech Católica
}

Juan Roger Rodríguez Ruíz*

\begin{abstract}
RESUMEN
E objetivo de la presente investigación fue determinar el nivel de cumplimiento de los principios de la Doctrina Social de la Iglesia (DSI) en el Proyecto Educativo Institucional (PEI) de la Universidad Católica Los Ángeles de Chimbote (Uladech Católica) según la percepción de la comunidad educativa. La investigación es básica, descriptiva, propositiva y cuantitativa. La población estuvo integrada por 42085 miembros de la comunidad universitaria, cuya información fue obtenida a través de la encuesta validada por expertos. Se determinó que el 67 \% de la comunidad universitaria está de acuerdo con que los principios de la DSI estén presentes en el PEI de la Uladech; el $84 \%$ manifiesta que el principio más aceptado es el «bien común» porque está presente en la misión de la universidad, los sílabos, los planes de aprendizaje y eventos académicos de responsabilidad social. Esto repercute en la mejora del proceso de comprensión de estos principios y en una propuesta más objetiva de un PEI con conceptos y categorías que respondan a las exigencias de una formación integral como personas responsables, profesionales competentes y cristianos comprometidos.
\end{abstract}

Palabras clave: Doctrina Social de la Iglesia, principios, proyecto educativo institucional

\section{ABSTRACT}

The objective of this research was to determine the level of compliance with the principles of the Social Doctrine of the Church (DSI) in the Institutional Education Project (PEI) of the Universidad Los Ángeles de Chimbote (Uladech Católica) as perceived by the education community. The research is basic descriptive and quantitative proactive. The population consisted of 42.085 members of the university community, whose information was obtained through the survey validated by experts. It was determined

* Doctor en Educación en Universidad Nacional de Educación a Distancia, España. Docente principal de la Universidad Católica Los Ángeles de Chimbote, Perú. Correo electrónico: qahais@yahoo.com.ar 
that $67 \%$ of the university community agrees that the principles of the DSI are present in the Uladech PEI; $84 \%$ say that the accepted principle is the "common good" because it is present in the mission of the university syllabi, learning plans and academic events Social Responsibility. This results in improved process understanding of these principles and in a more objective proposal of a PEI with concepts and categories that meet the requirements of a comprehensive education as responsible, competent professionals and committed Christians.

Keywords: Social Doctrine of the Church, principles, institutional educational project

\section{INTRODUCCIÓN}

La presente investigación se enmarca en la importancia sobre los principios y valores de la formación integral de los estudiantes universitarios. Determina cuál es el nivel de inclusión de los principios de la Doctrina Social de la Iglesia (DSI) en el Proyecto Educativo Institucional (PEI) de la Universidad Católica Los Ángeles de Chimbote.

A nivel internacional la Universidad Católica afronta nuevos retos que exigen, desde una antropología cristina, dar respuesta a los problemas que aquejan al mundo. En América Latina, la Universidad Católica tiene una presencia cultural considerable que contribuye al desarrollo social de los países. En Perú, la Universidad Católica ha contribuido notablemente en la formación de los líderes políticos y sociales y ha creado una línea de pensamiento basada en los principios de la DSI orientados en la formación de los nuevos profesionales. Se justifica esta investigación, porque el PEI de la Universidad (Rectorado, 2014) ha experimentado un cambio en su denominación como universidad católica; sin embargo, su aplicación es insuficiente y poco significativa en la asimilación de la comunidad universitaria.

Ciertamente, la universidad está en un proceso de permanente cambio; no obstante, no se ha logrado entender el significado de la mística y la vivencia como centro de estudios superior que debe estar impregnado por la Doctrina Social de la Iglesia.

La relevancia social del estudio ayudará a involucrar el espíritu de la DSI en el PEI. Asimismo, demostrará la validez de la DSI como línea transversal en los planes curriculares y vincular sus principios permanentes y valores fundamentales. También ayudará a formular, en el ámbito práctico, reflexivo y teórico, propuestas en el contenido de la DSI en el PEI y los planes curriculares y que los destinatarios logren entender y vivenciar los principios y valores de la DSI contenidos en el PEI, a fin de formar profesionales competentes, ciudadanos responsables y cristianos comprometidos.

El papa Francisco (2015) en la encíclica «Laudato si», declara: «El amor social es la clave de un auténtico desarrollo: Para plasmar una sociedad más humana, más digna de la persona, es necesario revalorizar el amor en la vida social -a nivel político, económico, cultural-, haciéndolo la norma constante y suprema de la acción» (p. 174).

En circunstancias adversas, Pío XII, con su autoridad moral y su prestigio, llevó la luz de la sabiduría cristiana a un número incontable de hombres de toda categoría y nivel social (Congregación para la Educación Católica, 1988). 
En referencia a la DSI, ya el papa Pablo VI (1967) en la encíclica «Popolurum progressio» afirma que «El desarrollo es el nuevo nombre de la paz», que puede ser considerada como una ampliación del capítulo sobre la vida económico-social de «Gaudium et spes» (1965).

Más recientemente, Juan Pablo II (1987) en la encíclica «Solicitudo rei socialis», estableció que la «Doctrina Social de la Iglesia no es una ideología, sino que pertenece a la teología moral» (p. 41). Al respecto, Stefano Fontana (2011), sostiene que Juan Pablo II: «en la Carta Apostólica Tertio millennio adveniente pidió a los católicos hacer un examen de conciencia acerca de la manera cómo han vivido y encarnado la Doctrina Social de la Iglesia» (p. 2).

En este sentido, Parma (2013) considera que la DSI enseña a que cada persona es un ser en relación, nacido para la comunión en la verdad y la caridad; y Blas Cerna (2013) sostiene que la formación profesional influye significativamente en la vocación del servicio de los docentes de educación religiosa. Así, la Universidad Católica está llamada a formar a la persona humana en los principios y valores humanos y en las virtudes cristianas.

Siguiendo esta perspectiva, Invernizzi (2013) sostiene que la enseñanza social de la Iglesia realizó un gran esfuerzo para ofrecer luces sobre las diferentes reconfiguraciones sociales, políticas, económicas y culturales en el mundo occidental. En este contexto, Benedicto XVI (2005) declara que «La justicia es el objeto y, por tanto, también la medida intrínseca de toda política. La política es más que una simple técnica para determinar los ordenamientos públicos: su origen y su meta están precisamente en la justicia, y esta es de naturaleza ética» (p. 28).

Desde esta realidad se plantea el problema en los siguientes términos: ¿Cuál es el nivel de inclusión de los principios de la Doctrina Social de la Iglesia en el Proyecto Educativo Institucional en la Universidad Católica Los Ángeles de Chimbote? El objetivo del estudio fue determinar el nivel de inclusión de los principios de la Doctrina Social de la Iglesia en el Proyecto Educativo Institucional de la Universidad Católica Los Ángeles de Chimbote.

\section{MATERIALES Y MÉTODOS}

Esta investigación, por su finalidad, es aplicada; por su contrastación, es descriptiva propositiva y, por el enfoque metodológico, es cuantitativa porque tiene una concepción lineal entre los elementos que conforman el problema (Hurtado y Toro, 1998). La población fue de 42085 miembros de la comunidad universitaria, integrada por 38812 estudiantes, 1379 egresados, 1327 docentes y 567 administrativos.

Se utilizó la técnica de la encuesta a través del cuestionario para estudiantes, egresados, docentes y administrativos, validado por expertos. Se buscó un entendimiento en profundidad, más que de exactitud, siguiendo el método inductivo que tiene una perspectiva holística porque considera esta realidad como un todo.

Se organizó y presentó la información tomando como base tablas de distribución de frecuencias, absolutas, simples y relativas porcentuales con respecto a los principios de la 
DSI. Como prueba estadística para contrastar la hipótesis se utilizó prueba de hipótesis de la proporción poblacional y los aplicadores informáticos SPSS, Versión 21 y Excel 2013.

\section{RESULTADOS}

Los resultados obtenidos de la muestra estudiada son los siguientes:

Tabla 1. Nivel de cumplimiento de los principios de la Doctrina Social de la Iglesia en el Proyecto Educativo Institucional de la Uladech Católica, en la percepción de los docentes, administrativos, estudiantes y egresados.

\begin{tabular}{|c|c|c|c|c|c|c|c|c|c|c|}
\hline Escala & $\begin{array}{c}\text { Dignidad } \\
\text { humana }\end{array}$ & $\%$ & Subsidiariedad & $\%$ & $\begin{array}{l}\text { Bien } \\
\text { común }\end{array}$ & $\%$ & Solidaridad & $\%$ & Total & $\%$ \\
\hline Desacuerdo & 0 & 0 & 13 & 4 & 1 & 0 & 1 & 0 & 15 & 1 \\
\hline $\begin{array}{l}\text { Poco de } \\
\text { acuerdo }\end{array}$ & 43 & 12 & 18 & 5 & 22 & 6 & 47 & 12 & 130 & 9 \\
\hline $\begin{array}{l}\text { Medianamente } \\
\text { de acuerdo }\end{array}$ & 51 & 14 & 30 & 8 & 61 & 17 & 53 & 14 & 342 & 23 \\
\hline De acuerdo & 127 & 35 & 193 & 52 & 140 & 38 & 133 & 35 & 598 & 40 \\
\hline $\begin{array}{l}\text { Totalmente de } \\
\text { acuerdo }\end{array}$ & 145 & 40 & 117 & 32 & 143 & 39 & 146 & 38 & 399 & 27 \\
\hline Total & 366 & 100 & 371 & 100 & 367 & 100 & 380 & 100 & 1484 & 100 \\
\hline
\end{tabular}

Fuente: Fichas de registro sobre los principios de la DSI en el Proyecto Educativo Institucional

En relación al nivel de cumplimiento de los principios de la Doctrina Social de la Iglesia presentes en el Proyecto Educativo Institucional de la Uladech Católica, en la percepción de los docentes, administrativos, estudiantes y egresados, se registró lo siguiente:

El $1 \%$ está en desacuerdo, el $9 \%$ poco de acuerdo y el $23 \%$ medianamente de acuerdo, sin embargo, el $40 \%$ está de acuerdo y el $27 \%$ está totalmente de acuerdo con los principios de la Doctrina Social de la Iglesia en el Proyecto Educativo Institucional en la Uladech Católica. De modo que la comunidad universitaria (67\%) si cumple con los principios de la Doctrina Social de la Iglesia presentes en el Proyecto Educativo Institucional de la Uladech Católica.

Tabla 2. El principio de la Doctrina Social de la Iglesia privilegiado en el Proyecto Educativo Institucional en la percepción de los docentes, administrativos, estudiantes y egresados.

\begin{tabular}{|c|c|c|c|c|c|c|c|c|c|c|}
\hline Escala & $\begin{array}{l}\text { Dignidad } \\
\text { humana }\end{array}$ & $\%$ & $\begin{array}{c}\text { Bien } \\
\text { común }\end{array}$ & $\%$ & Subsidiariedad & $\%$ & Solidaridad & $\%$ & Total & $\%$ \\
\hline Desacuerdo & 0 & 0 & 13 & 4 & 1 & 0 & 1 & 0 & 15 & 1 \\
\hline Poco de acuerdo & 43 & 12 & 18 & 5 & 22 & 6 & 47 & 12 & 130 & 9 \\
\hline $\begin{array}{l}\text { Medianamente } \\
\text { de acuerdo }\end{array}$ & 51 & 14 & 30 & 8 & 61 & 17 & 53 & 14 & 342 & 23 \\
\hline De acuerdo & 127 & 35 & 193 & 52 & 140 & 38 & 133 & 35 & 598 & 40 \\
\hline $\begin{array}{l}\text { Totalmente de } \\
\text { acuerdo }\end{array}$ & 145 & 40 & 117 & 32 & 143 & 39 & 146 & 38 & 399 & 27 \\
\hline Total & 366 & 100 & 371 & 100 & 367 & 100 & 380 & 100 & 1484 & 100,0 \\
\hline
\end{tabular}

Fuente: Fichas de registro sobre el nivel de cumplimiento de los principios de la Doctrina Social de la Iglesia en el Proyecto Educativo Institucional 
En relación al principio de la Doctrina Social de la Iglesia privilegiado en el Proyecto Educativo Institucional en la percepción de los docentes, administrativos, estudiantes y egresados, se registró lo siguiente:

El principio de la solidaridad tiene el $73 \%$, la dignidad humana el $75 \%$ y la subsidiariedad el $77 \%$, sin embargo, el principio del bien común tiene $84 \%$.

De modo que el principio de la Doctrina Social de la Iglesia privilegiado en el Proyecto Educativo Institucional en la percepción de los docentes, administrativos, estudiantes y egresados es el «bien común», con $84 \%$.

Tabla 3. Nivel de cumplimiento de los principios de la Doctrina Social de la Iglesia en el Proyecto Educativo Institucional de administrativos con egresados y de estudiantes con docentes.

\begin{tabular}{|c|c|c|c|c|c|c|c|c|c|c|}
\hline \multirow[t]{2}{*}{ Escala } & \multicolumn{2}{|c|}{ Administrativos } & \multicolumn{2}{|c|}{ Egresados } & \multicolumn{2}{|c|}{ Estudiantes } & \multicolumn{2}{|c|}{ Docentes } & \multicolumn{2}{|c|}{ Total } \\
\hline & $\mathrm{N}^{0}$ & $\%$ & $\mathrm{~N}^{\mathrm{o}}$ & $\%$ & $\mathrm{~N}^{0}$ & $\%$ & $\mathrm{~N}^{\mathrm{o}}$ & $\%$ & $\mathrm{~N}^{0}$ & $\%$ \\
\hline Desacuerdo & 0 & 0 & 2 & 4 & 13 & 1 & 0 & 0 & 15 & 1 \\
\hline Poco acuerdo & 4 & 20 & 11 & 22 & 104 & 8 & 11 & 23 & 130 & 9 \\
\hline Medianamente de acuerdo & 9 & 45 & 20 & 41 & 147 & 11 & 19 & 40 & 195 & 13 \\
\hline De acuerdo & 6 & 30 & 15 & 31 & 555 & 41 & 17 & 36 & 593 & 40 \\
\hline Totalmente de acuerdo & 1 & 5 & 1 & 2 & 549 & 40 & 0 & 0 & 551 & 37 \\
\hline Total & 20 & 100 & 49 & 100 & 1368 & 100 & 47 & 100 & 1484 & 100,00 \\
\hline
\end{tabular}

Fuente: Fichas de registro sobre el nivel de cumplimiento de los principios de la Doctrina Social de la Iglesia en el Proyecto Educativo Institucional

La proporción de administrativos (45\%) que están medianamente de acuerdo es mayor que el de egresados (41\%) respecto al nivel de cumplimiento de los principios de la Doctrina Social de la Iglesia en el Proyecto Educativo Institucional.

La proporción de estudiantes (41\%) que están de acuerdo es mayor que en docentes (36\%) respecto al nivel de cumplimiento de los principios de la Doctrina Social de la Iglesia en el Proyecto Educativo Institucional.

Se desea probar la hipótesis:

H1: La proporción de administrativos que están de medianamente de acuerdo es mayor que en egresados respecto al nivel de cumplimiento de los principios de la Doctrina Social de la Iglesia en el Proyecto Educativo Institucional.

Como Zp = 0.3039,6044 < 1.6449 pertenece a la región de aceptación. Aceptamos Ho. Por lo tanto, no existe diferencia significativa entre la proporción de administrativos y egresados que están medianamente de acuerdo respecto al nivel de cumplimiento de los principios de la Doctrina Social de la Iglesia en el Proyecto Educativo Institucional.

Se ha considerado un nivel de significación de 5\%. 


\section{DISCUSIÓN}

En relación con el nivel de cumplimiento de los principios de la Doctrina Social de la Iglesia presentes en el Proyecto Educativo Institucional de la Uladech Católica, en la percepción de los docentes, administrativos, estudiantes y egresados (Tabla 1), se registró lo siguiente:

El $1 \%$ está en desacuerdo, el $9 \%$ poco de acuerdo y el $23 \%$ medianamente de acuerdo, sin embargo, el $40 \%$ está de acuerdo y el $27 \%$ está totalmente de acuerdo con los principios de la Doctrina Social de la Iglesia en el Proyecto Educativo Institucional en la Uladech Católica.

En forma general, se registró que el 67 \% manifiesta que sí se cumplen los principios de la Doctrina Social de la Iglesia en la elaboración del Proyecto Educativo Institucional de la Uladech Católica.

Al respecto, Aparicio (2012) propone retomar el referente moral de la Doctrina Social de la Iglesia que aporta a través de sus principios generales, normas de juicio y, sobre todo, pautas prácticas de acción.

El papa Francisco (2015) declara que «El amor social es la clave de un auténtico desarrollo: Para plasmar una sociedad más humana, más digna de la persona, es necesario revalorizar el amor en la vida social -a nivel político, económico, cultural-, haciéndolo la norma constante y suprema de la acción» (p. 174).

La aceptación mayoritaria de la comunidad universitaria respecto a la Doctrina Social de la Iglesia expresa la intencionalidad de conocer con mayor profundidad sus principios en el ámbito universitario, tal como se recomienda en el Documento de Aparecida y en los planes estratégicos del Departamento de Justicia y Solidaridad, así como la valoración de la Oducal (organismo coordinador de las Universidades Católicas Latinoamericanas) y del Pontificio Consejo Justicia y Paz para quienes los proyectos de futuro suponen realizaciones concretas y compromisos para la difusión del Compendio de Doctrina Social de la Iglesia y una puerta abierta para la Nueva Evangelización en la universidad.

En conclusión, la comunidad universitaria está de acuerdo que los principios de la Doctrina Social de la Iglesia estén presentes en la elaboración del Proyecto Educativo Institucional de la Uladech Católica.

En relación con el principio de la Doctrina Social de la Iglesia privilegiado en el Proyecto Educativo Institucional (Tabla 2) en la percepción de los docentes, administrativos, estudiantes y egresados es el «bien común», con $84 \%$.

Continúa el principio de la dignidad humana con $75 \%$, la subsidiariedad con $77 \%$ y con menor proporción la solidaridad con $73 \%$.

Este resultado refleja que la comunidad universitaria está mejor sensibilizada en el principio del «bien común» que aparece en la misión de la universidad, en los sílabos y eventos académicos. 
Parma (2013) centra su análisis en conceptos políticos insertos en la Doctrina Social de la Iglesia y aspira a la construcción del «bien común», lo que debería ser la praxis política. En este sentido, la Doctrina Social de la Iglesia puede producir hoy el mismo efecto en nuestro contexto actual que Pablo VI, en su testamento, calificó de «dramático y triste, y sin embargo magnífico». Así, los principios de la Doctrina Social pueden convertirse para el cristiano de nuestro tiempo, en una fuente de orientación y un instrumento genuino de evangelización (Nguyen, 2003).

Al respecto, Souto Coelho (1995) sostiene que la Doctrina Social de la Iglesia tiene como centro la dignidad de la persona humana y busca defenderla y dar principios que ayuden a su crecimiento y desarrollo. En este contexto, la enseñanza social es parte esencial del mensaje cristiano porque educa a las nuevas generaciones en un tipo de hombre y contribuye a la construcción de una sociedad más justa y solidaria.

En conclusión, el principio de la Doctrina Social de la Iglesia privilegiado en el Proyecto Educativo Institucional de la Universidad Católica Los Ángeles de Chimbote, es el «bien común» (84 \%) porque ha sido mejor presentado en la misión de la universidad, en los sílabos y planes de aprendizaje (SPA) y los eventos académicos y de responsabilidad social.

En cuanto a la proporción de administrativos con egresados y de estudiantes con docentes (Tabla 3) respecto al nivel de cumplimiento de los principios de la Doctrina Social de la Iglesia en el Proyecto Educativo Institucional, los resultados son los siguientes:

La proporción de administrativos (45\%) que están «medianamente de acuerdo» es mayor que en egresados (41\%) respecto al nivel de cumplimiento de los principios de la Doctrina Social de la Iglesia en el Proyecto Educativo Institucional.

La proporción de estudiantes (41\%) que están «de acuerdo» es mayor que en docentes (36\%) respecto al nivel de cumplimiento de los principios de la Doctrina Social de la Iglesia en el Proyecto Educativo Institucional.

Resulta interesante verificar cómo los administrativos, que contribuyen al proceso educativo desde la gestión, y los estudiantes que vienen a aprender y que muestran una actitud de apertura, búsqueda y receptividad, tienen una mejor percepción sobre el cumplimiento de los principios de la Doctrina Social de la Iglesia en el Proyecto Educativo Institucional respecto a los docentes que vienen a impartir y enseñar lo que saben y a los egresado que consideran que ya aprendieron y están listos para asumir los retos de la sociedad.

Se considera que esto obedece a la actitud de los actores; mientras los administrativos y estudiantes se muestran en actitud alerta y abiertos al aprendizaje, los docentes y egresados se muestran conocedores y experimentados.

De esto se concluye que la proporción de los administrativos y estudiantes es mayor respecto a la de los egresados y docentes sobre el cumplimiento de los principios de la Doctrina Social de la Iglesia en el Proyecto Educativo Institucional de la Universidad Católica Los Ángeles de Chimbote. 
Los resultados indican que los principios de la DSI iluminan a la persona y la orientan hacia su realización personal y comunitaria. Al respecto, Escobar (2012) sostiene que el desarrollo de la DSI muestra claramente que ella misma no es una ideología cristiana, sino una teología moral social cuyos fundamentos están en el Evangelio, el magisterio de la Iglesia y la tradición apostólica. Asimismo, la fe, en interacción con la razón, busca aplicar en una sociedad siempre cambiante, el mensaje de Jesús a fin de que la sociedad refleje más fielmente el plan de Dios para la humanidad.

Así, la comunidad universitaria expresa su aceptación y reconocimiento sobre los principios de la DSI y desea comprender el significado de la fe y la cultura como realidades autónomas. Además de constatar que la fe necesita de la mediación cultural para poder expresarse, quiere afrontar el reto que supone la ruptura entre la fe y la cultura en el momento presente y apreciar el potencial de la fe cristiana para evangelizar la Universidad Católica, encarnando los valores cristianos.

En conclusión, los miembros de la comunidad académica perciben mayoritariamente (67 \%) que están «de acuerdo» con que los principios de la DSI están presentes en el PEI, es decir, el nivel de inclusión de estos principios es considerable, aunque a la vez se aspira a verificar su cumplimiento.

\section{REFERENCIAS BIBLIOGRÁFICAS}

Aparicio, A. (2010). Aportes de la Doctrina Social de la Iglesia para una deontología del consumo. Revista Iberoamericana de Teología, 6(11): pp. 31-54.

Benedicto XVI. (2005). Deus caritas est. Ciudad del Vaticano: Libreria Editrice Vaticana.

Blas Cerda, C. P. (2013). La formación profesional cristiana y la vocación de servicio de los docentes de educación religiosa de la oficina diocesana de educación católicaHuánuco. Revista de Investigación en Psicología, 16(2): pp. 139-155.

Congregación para la Educación Católica. (1988). Orientaciones para el estudio y enseñanza de la doctrina social de la Iglesia en la formación de los sacerdotes. Ciudad del Vaticano: Tipografía Poliglota Vaticana.

Escobar Delgado, R. A. (2012). La Doctrina Social de la Iglesia: Fuentes y principios. Prolegómenos. Derechos y Valores, 15(30): pp. 99-117.

Fontana, S. (2011) El papa Juan Pablo II y la Doctrina Social de la Iglesia. Una mirada a su gran pontificado. Recuperado de http://www.vanthuanobservatory.org/notizie-dsc/ notizia-dsc.php?lang=es\&id $=1163$

Francisco. (2015). Encíclica Laudato si. Ciudad del Vaticano: Tipografía Vaticana.

Hurtado, I. y Toro, G. (2001). Paradigmas y Métodos de Investigación en Tiempos de Cambio (4. ${ }^{a}$ ed.). Episteme: Valencia-Venezuela.

Invernizzi, M. (2013). 1891-1991. Cien años de Doctrina Social: entre proyecto, eclipse y continuidad. Boletín de DSI, 6(16): pp. 12-18. 
Juan Pablo II. (1987). Solicitudo Rei Socialis. Ciudad del Vaticano: Libreria Editrice Vaticana.

Pablo VI. (1967). Carta encíclica. Populorum progressio. Ciudad del Vaticano: Libreria Editrice Vaticana.

Parma, E. (2013). Política en la Doctrina Social de la Iglesia. Universum [online]. 2013, 28(1): pp. 231-245.doi: org/10.4067/S0718.

Pontificio Consejo Justicia y Paz. (2005). Compendio de la Doctrina Social de la Iglesa. Ciudad del Vaticano: Libreria Editrice Vaticana.

Rectorado. (2014). Proyecto Educativo Institucional. Chimbote: Universidad Católica Los Ángeles de Chimbote.

Souto Coelho, J. (1995). Iniciación a la Doctrina Social de la Iglesia. Madrid: San Pablo. 